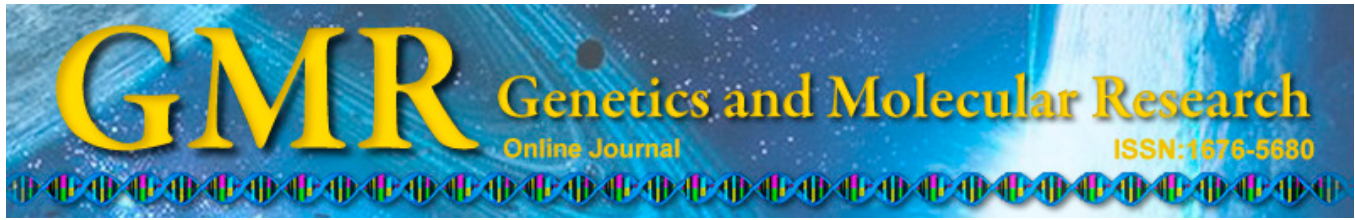

\title{
Bioequivalence of clopidogrel hydrogen sulfate tablets in healthy Chinese volunteers
}

\author{
Y.G. Yang, M. Zhang, N. Jiang, L.X. Song, X.T. Xu, X.H. Di, L. Xu, \\ J. Xu and G.T. Zhao \\ Department of Pharmacology, General Hospital of Beijing Military Command, \\ Beijing, China \\ Corresponding author: M. Zhang \\ E-mail: zhangmei_yang6@sina.com \\ Genet. Mol. Res. 14 (4): 16736-16743 (2015) \\ Received August 26, 2015 \\ Accepted October 29, 2015 \\ Published December 14, 2015 \\ DOI http://dx.doi.org/10.4238/2015.December14.1
}

\begin{abstract}
We aimed to evaluate the bioequivalence of clopidogrel in healthy Chinese volunteers after administration of a single oral dose. We administered a single oral dose of $75 \mathrm{mg}$ clopidogrel (test and reference) to 32 healthy Chinese volunteers according to an open, randomized, crossover design. The concentration of clopidogrel acid (carboxylic metabolite of clopidogrel) in the plasma was determined using liquid chromatography-tandem mass spectrometry (LC-MS/MS). Bioequivalence of the test and reference preparations were calculated using analysis of variance and one-sided $t$-test by using the DAS 2.0 software. The pharmacokinetic parameters of the test and reference preparations were as follows: peak plasma concentration $\left(\mathrm{C}_{\max }\right)$, $1351.101 \pm 654.955 \mathrm{ng} / \mathrm{mL}$ and $1184.652 \pm 607.713 \mathrm{ng} / \mathrm{mL}$; area under the curve, $2642.017 \pm 1093.848 \mathrm{ng} \cdot \mathrm{h} / \mathrm{mL}$ and $2780.666 \pm 1283.100$ $\mathrm{ng} \cdot \mathrm{h} / \mathrm{mL}$; and time to reach $\mathrm{C}_{\max }\left(\mathrm{T}_{\max }\right), 0.789 \pm 0.318 \mathrm{~h}$ and $0.953 \pm$ $0.633 \mathrm{~h}$, respectively. The relative bioavailability of the formulation was $101.7 \pm 35.3 \%$, which indicated that the test preparation was bioequivalent to the reference drug.
\end{abstract}

Key words: Clopidogrel; Clopidogrel acid; Bioequivalence 


\section{INTRODUCTION}

Clopidogrel is an inhibitor of platelet aggregation that inhibits the combination of adenosine diphosphate (ADP) with its platelet receptor, and subsequently, inhibits the activation of glycoprotein GPIIb/IIIa induced by ADP (Stanisstreet and Smith, 1977). The specific pharmacological target of clopidogrel is the ADP-induced platelet activation process. A previous study has shown that clopidogrel is inactive in vitro, and a biotransformation by the liver is required for clopidogrel to exert its antiaggregating activity (Savi et al., 1992). Therefore, clopidogrel can be considered as a precursor of an active metabolite. The major metabolite of clopidogrel is inactive clopidogrel carboxylic acid (Pereillo et al., 2002).

The objective of this study was to identify the bioequivalence of clopidogrel hydrogen sulfate tablets in healthy Chinese volunteers. Thus, we determined the concentration of clopidogrel carboxylic acid using liquid chromatography-tandem mass spectrometry (LC-MS/MS) to explain the characteristics of drug metabolism in Han Chinese subjects. Subsequently, we evaluated the bioavailability of clopidogrel tablets and examined the bioequivalence between clopidogrel and the reference drug.

\section{MATERIAL AND METHODS}

We performed our study at the General Hospital of Beijing Military Command, Beijing, China, between March 2012 and July 2012. Signed informed consent was obtained from all the volunteers. Our study was approved by the Ethics Committee in Beijing Military Region General Hospital.

\section{Drugs and reagents}

We used clopidogrel bisulfate tablets (Areplex; Adelman Pharmaceutical Co., Ltd., Poland) as the test drug. Each tablet contained $75 \mathrm{mg}$ clopidogrel. We used clopidogrel bisulfate tablets (Plavix; Sanofi Pharma and Bristol-Myers Squibb, France) containing $75 \mathrm{mg}$ clopidogrel as the reference drug. Clopidogrel acid reference substance (content: $98 \%$, batch No. 144457-28-3, product No. C587240) was purchased from Toronto Research Chemicals Inc. (North York, Canada). Simvastatin (content: 99.3\%, batch No. 100601-200301) was purchased from the National Institute for the Control of Pharmaceutical and Biological Products (Beijing, China) and was used as the internal standard.

\section{Conditions of chromatography and mass spectrometry}

THERMO Basic- 8 column $(2.1 \times 100 \mathrm{~mm}, 5 \mu \mathrm{m})$ manufactured by Thermo Electron Corporation was used for LC. Acetonitrile-water (containing $0.1 \%$ formic acid, 70:30) was used as the mobile phase. The flow rate was $0.2 \mathrm{~mL} / \mathrm{min}$. We added $10 \mu \mathrm{L}$ of the sample into the column at a temperature of $25^{\circ} \mathrm{C}$. For MS, the spray voltage was set at $4000 \mathrm{~V}$. The flow rate of the sheath gas was 5 arb and that of the auxiliary gas was 20 arb. The capillary temperature was $270^{\circ} \mathrm{C}$. Additionally, the collision energies of clopidogrel acid and the internal standard simvastatin were 26 and $24 \mathrm{EV}$, respectively. We used the selective reaction monitoring as the scan mode for MS. The $\mathrm{m} / \mathrm{z}$ values of the ions tested were clopidogrel acid $[\mathrm{M}+\mathrm{H}]^{+}$, $\mathrm{m} / \mathrm{z} 308 \rightarrow 198$ and internal standard simvastatin $[\mathrm{M}+\mathrm{H}]^{+}, \mathrm{m} / \mathrm{z} 441 \rightarrow 325$. The scan interval was set at $0.2 \mathrm{~s}$. 


\section{Plasma sample handling}

We transferred $100 \mu \mathrm{L}$ plasma sample into a $1.5 \mathrm{~mL}$ centrifuge tube. Then, we added 10 $\mu \mathrm{L}$ internal standard solution ( $50 \%$ methanol solution of simvastatin, $1 \mu \mathrm{g} / \mathrm{mL}$ ). Subsequently, we added $400 \mu \mathrm{L}$ methanol and vortexed the sample for $2 \mathrm{~min}$. The mixture was centrifuged at $10,000 \mathrm{rpm}$ for $10 \mathrm{~min}$, and the supernatant $(100 \mu \mathrm{L})$ was transferred into a sampler vial.

\section{Selection of subjects}

We enrolled 32 healthy Chinese volunteers (men; mean age, $25.7 \pm 3.0$ years). The inclusion criteria included no reported abnormities in physical and laboratory examination before the test, no participation in any clinical drug trials within the last 2 months, and no administration of any drugs within last 3 months.

\section{Experimental design}

The volunteers were randomly divided into two groups: group A and group B. Subjects in group A received $75 \mathrm{mg}$ test drug (Areplex; A) in the first cycle, and $75 \mathrm{mg}$ reference drug (Plavix; R) in the second cycle. Volunteers in group B received $75 \mathrm{mg}$ test drug $(\mathrm{R})$ in the first cycle, and $75 \mathrm{mg}$ reference drug (A) in the second cycle. The interval between the two cycles was 7 days. Venous blood $(2.5 \mathrm{~mL})$ was collected before drug administration and $0.25,0.5,0.75$, $1,1.25,1.5,2,4,8,12$, and $24 \mathrm{~h}$ after drug administration. Heparin anticoagulant tubes were used to collect blood samples, and the exact time of blood collection was recorded in individual case report forms. After blood collection, blood samples were immediately centrifuged at 3000 $\mathrm{rpm}$ for $10 \mathrm{~min}\left(4^{\circ} \mathrm{C}\right)$. The separated plasma was transferred to a $1.5 \mathrm{~mL}$ centrifuge tube. Subsequently, the plasma samples were stored at $-70^{\circ} \mathrm{C}$ for further analysis.

\section{Statistical analysis}

DAS 2.0 (general program for bioequivalence study data processing developed by Wannan Medical College, Wuhu, China) was adopted to calculate pharmacokinetic parameters and for statistical analysis. After the main pharmacokinetic parameters were logarithmically transformed, variance analysis, two one-sided $t$-test, and $90 \%$ confidence interval were determined for the peak concentration $\left(\mathrm{C}_{\max }\right)$ and the area under the concentration-time curve $\left(\mathrm{AUC}_{0-\mathrm{t}}\right.$, trapezoidal method). The Wilcoxon nonparametric test was performed on the time to peak concentration ( $\mathrm{T}_{\max }$, actually measured value) to check whether the test drug and the reference drug were bioequivalent. The relative bioavailability was calculated as follows:

$\mathrm{F}=\mathrm{AUC}_{\mathrm{T}} / \mathrm{AUC}_{\mathrm{R}} \times 100 \%$, where $A U C_{\mathrm{T}}$ is the $\mathrm{AUC}$ of the test drug and $A U C_{\mathrm{R}}$ is the $\mathrm{AUC}$ of the reference drug.

\section{RESULTS}

\section{Specificity analysis}

Chromatograms of the blank plasma, blank plasma + clopidogrel acid standard sample + internal standard, and the plasma after oral administration of clopidogrel carboxylic acid 
are shown in Figure 1. Clopidogrel acid and the internal standard were well separated from impurities in the plasma, and the endogenous impurities caused no interference in the plasma chromatogram.

A

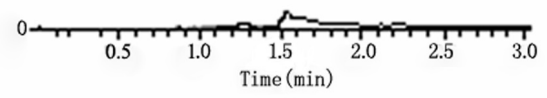

$1.04 \mathrm{I}$

B
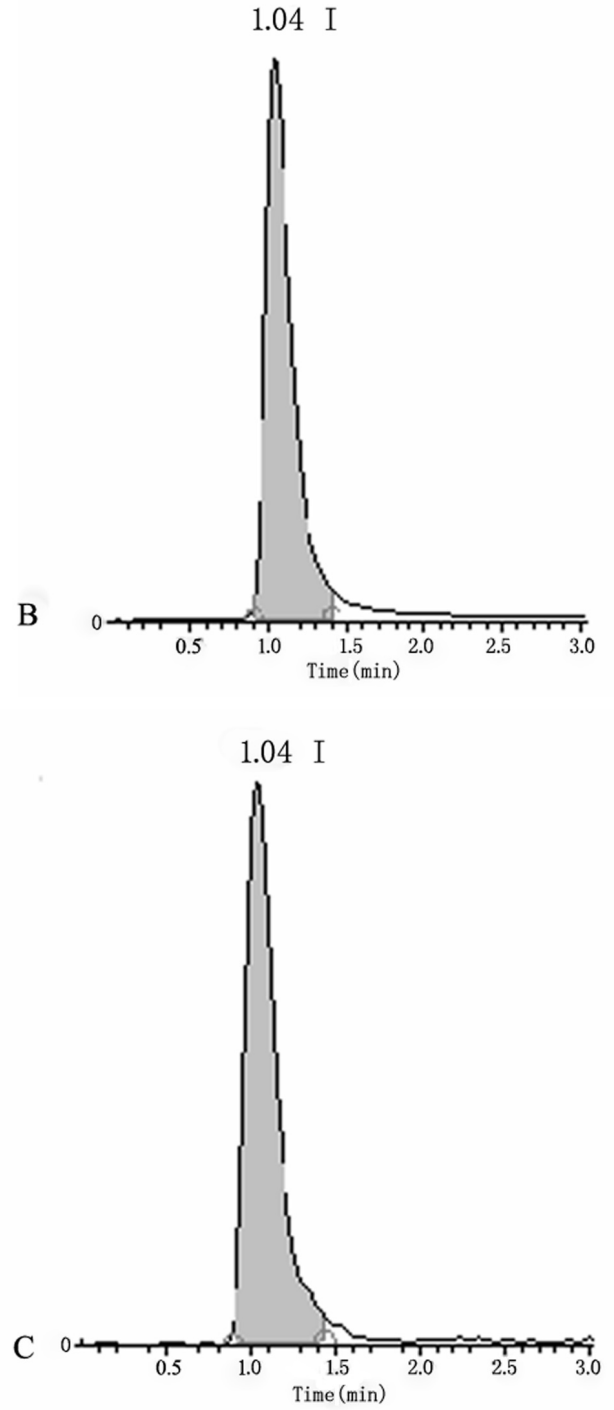

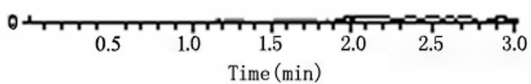

1.43 II

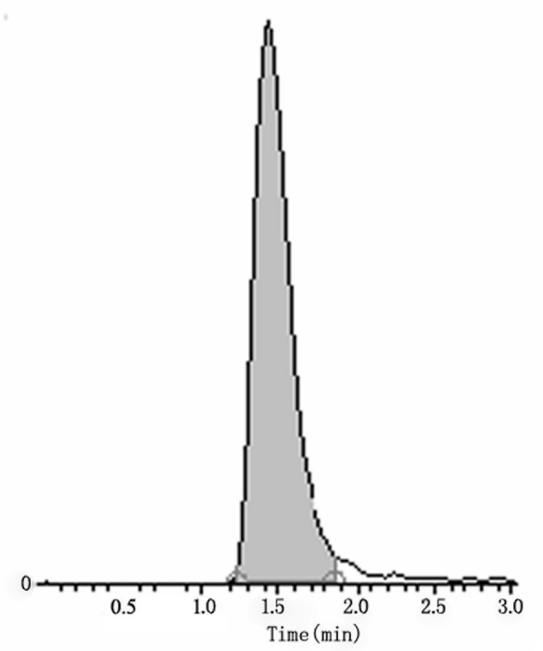

1.42 II

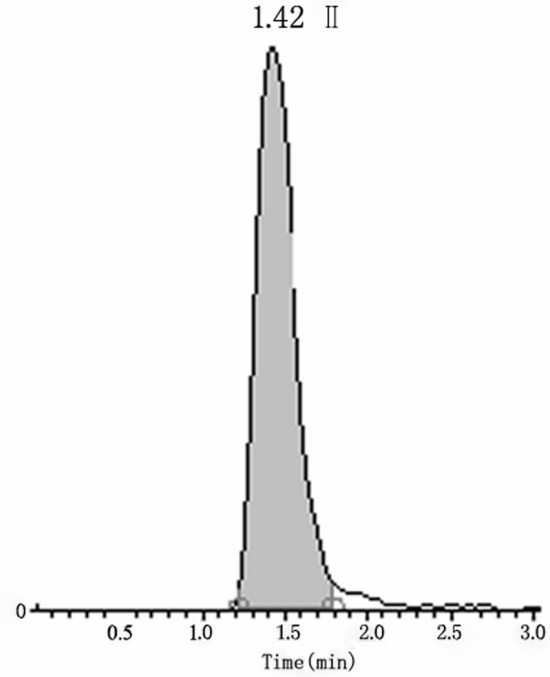

Figure 1. Chromatograms of clopidogrel carboxylic acid and the internal standard (simvastatin) in the plasma. I: Clopidogrel carboxylic acid; II: Internal standard (simvastatin). A = Blank plasma; $\mathbf{B}=$ blank plasma + clopidogrel acid standard sample $(3500 \mathrm{ng} / \mathrm{mL})+$ internal standard; $\mathbf{C}=$ plasma sample obtained from subject number 21 (obtained $1.25 \mathrm{~h}$ after oral administration of $75 \mathrm{mg}$ clopidogrel bisulfate tablets). 


\section{Linear relationship and the lower limit of quantification}

Clopidogrel carboxylic acid standard solution was added into blank plasma to prepare various concentrations of clopidogrel carboxylic acids $(10,50,100,250,500,1000,2000$, and $4000 \mathrm{ng} / \mathrm{mL}$ ). Weighted linear regression was performed as follows: the ratio between clopidogrel carboxylic acid concentration and the internal standard peak area was defined as the ordinate, while clopidogrel carboxylic acid concentration was set as the abscissa (weight: $1 / \mathrm{C}$ ). The results indicated a clear linear relationship between the concentration and peak area $[Y=0.0244486+0.0006043 X(R=0.9991)]$ when the level of plasma clopidogrel carboxylic acid ranged from 10 to $4000 \mathrm{ng} / \mathrm{mL}$. The lower limit of quantification was $10 \mathrm{ng} / \mathrm{mL}$ [relative standard deviation $(\mathrm{RSD})=5.44 \%, \mathrm{~N}=6]$.

\section{Precision and accuracy}

Clopidogrel carboxylic acid standard solution $(10 \mu \mathrm{L})$ was added into blank plasma $(100 \mu \mathrm{L})$ to prepare various concentrations of clopidogrel carboxylic acid $(25,500$, and 3500 $\mathrm{ng} / \mathrm{mL}$ ). The entire chromatography procedure was repeated for 3 consecutive days. Quality control (QC) samples were measured to calculate the accuracy and precision according to the standard curve on each day (Table 1). The intraday and interday precision (RSD) of QC samples at each concentration level was less than $15 \%$, and the accuracy ranged between 85 and $115 \%$.

$\begin{aligned} & \text { Table 1. Precision and accuracy of plasma clopidogrel carboxylic acid measured using liquid chromatography- } \\
& \text { tandem mass spectrometry (LC-MS/MS) }\end{aligned}$
\begin{tabular}{lccr}
\hline Concentration (ng/mL) & Accuracy (\%) & Precision [relative standard deviation (\%)] \\
\cline { 2 - 4 } & & Intraday & Interday \\
\hline 25 & 90.09 & 9.40 & 3.20 \\
500 & 97.43 & 6.46 & 13.00 \\
3500 & 94.00 & & 3.59 \\
\hline
\end{tabular}

\section{Extraction recovery and matrix effect}

Clopidogrel carboxylic acid standard solution was added into blank plasma to prepare samples of various concentrations $(25,500$, and $3500 \mathrm{ng} / \mathrm{mL})$. Concentration of the internal standard sample was set at $25 \mathrm{ng} / \mathrm{mL}$, and peak areas (A1) were obtained after the samples were analyzed.

Methanol precipitation reagent $(400 \mu \mathrm{L})$ was added into blank plasma, vortexed for $2 \mathrm{~min}$, and then, centrifuged at 10,000 rpm for $10 \mathrm{~min}$. The supernatant was placed into a 1.5 $\mathrm{mL}$ EP tube and dried at $37^{\circ} \mathrm{C}$ using nitrogen, and then, distilled water $(100 \mu \mathrm{L})$ was added. Subsequently, the precipitation reagent was added to clopidogrel carboxylic acid standard solution and the internal standard and vortexed for $30 \mathrm{~s}$. The supernatant $(10 \mu \mathrm{L})$ was tested to obtain various peak areas (A2). The extraction recovery of the 3 clopidogrel carboxylic acid concentrations was $87.9 \pm 6,86 \pm 2.9$, and $92.8 \pm 8.5 \%$, while that of the internal standard $(25 \mathrm{ng} / \mathrm{mL})$ was $89 \pm 11.5 \%$. The peak area ratio (A1/A2) was used to calculate the extraction recovery rate. The matrix effect was defined as $1-\mathrm{A} 2 / \mathrm{A} 3$, where $A 3$ was considered as peak area when the blank plasma was substituted by distilled water. The matrix effect of the 3 clopidogrel carboxylic acid concentrations was $4.3,1$, and $-0.1 \%$, while that of the internal 
standard was 3\%. Our results showed that clopidogrel carboxylic acid and the internal standard had high and stable extraction recovery rates, and the blood matrix had less matrix effects on clopidogrel carboxylic acid and the internal standard.

\section{Stability test}

QC samples with various clopidogrel carboxylic acid concentrations $(25,500$, and $3500 \mathrm{ng} / \mathrm{mL}$ ) were prepared in advance. The samples underwent 3 freeze/thaw cycles and were stored at $-70^{\circ} \mathrm{C}$ for 30 days for the stability test. The sample solution was placed in a liquid sample chamber for $24 \mathrm{~h}$, while the plasma sample was placed at room temperature for $2 \mathrm{~h}$. The results showed that the relative deviation of clopidogrel carboxylic acid at 3 concentrations under 4 different stability tests was between -9.1 and $9.6 \%$, which suggested that the favorable stability of clopidogrel carboxylic acid in plasma samples could meet the requirements of biological sample analysis.

\section{Plasma drug concentration-time curve}

The average plasma clopidogrel carboxylic acid concentration-time curve in the patients who received a single dose of oral A was similar to that of the R (Figure 2).

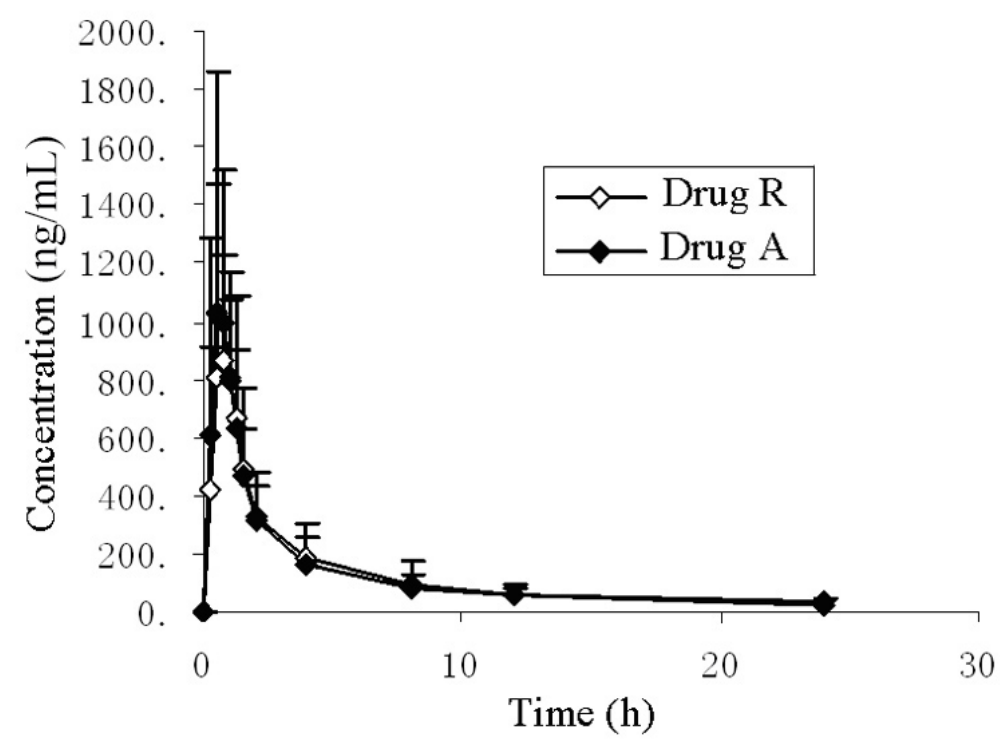

Figure 2. Average plasma drug concentration-time curve for Areplex (A) and Plavix (R) in healthy subjects.

\section{Pharmacokinetics parameters}

The DAS 2.0 software was used to analyze the plasma drug data after oral administration of $\mathrm{A}$ and $\mathrm{R}$ in 32 healthy subjects. The main pharmacokinetics parameters are shown in Table 2 . 
Table 2. Main pharmacokinetics parameters for the test and reference clopidogrel bisulfate tablets.

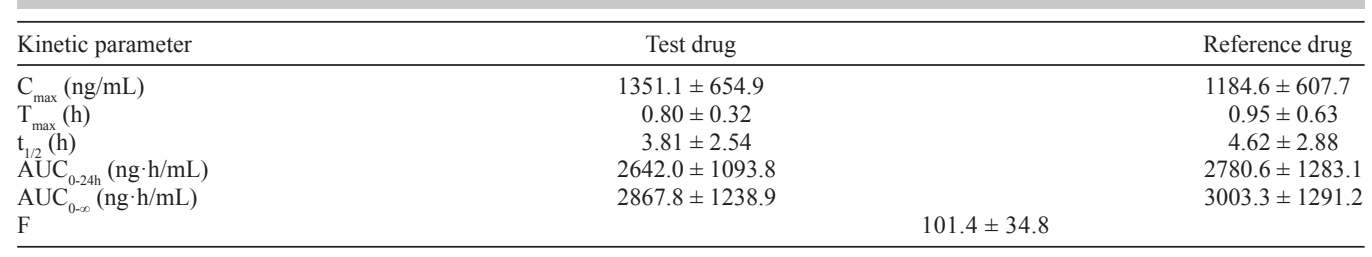

\section{Bioequivalence evaluation}

The average relative bioequivalence (A $v s \mathrm{R})$ was $101.4 \pm 34.8 \%$. Multivariate analysis of variance and two one-sided $t$-test were performed after log transformation of AUC and $\mathrm{C}_{\max }$ (Table 3). The AUC of A was 80 to $125 \%$ compared to that of Plavix, while $\mathrm{C}_{\max }$ of Areplex was 70 to $143 \%$ of that of Plavix. No significant difference was observed in the $\mathrm{T}_{\max }$ between Areplex and Plavix $(\mathrm{P}>0.05)$, which indicated that Areplex and Plavix were bioequivalent.

Table 3. The main pharmacokinetics parameters of Areplex (A) and Plavix (R) in 32 healthy subjects (T0.1 $(30)=1.697 ; * \mathrm{P}<0.05)$.

\begin{tabular}{lccc}
\hline Parameters & $\mathrm{T}_{1}$ & $\mathrm{~T}_{2}$ & $90 \%$ Confidence interval (CI) \\
\hline LnAUC $_{0-t}$ & $5.843^{*}$ & $2.560^{*}$ & $99.6-132.9$ \\
LnAUC $_{0-\infty}$ & $2.750^{*}$ & $4.356^{*}$ & $85.5-105.8$ \\
LnC $_{\max }$ & $2.860^{*}$ & $4.901^{*}$ & $85.5-104.0$ \\
\hline
\end{tabular}

\section{DISCUSSION}

In this study, Areplex or Plavix was orally administered to healthy subjects in accordance with a two-compartment pharmacokinetic model. Analysis of variance showed that $\mathrm{AUC}_{0-24 \mathrm{~h}}$ and $\mathrm{C}_{\max }$ of Areplex and Plavix were bioequivalent. Compared to results reported previously, our results showed a decrease in the AUC and $\mathrm{C}_{\max }$ in the healthy Chinese subjects (Junior et al., 2010), while the values of $\mathrm{t}_{1 / 2}$ and $\mathrm{T}_{\max }$ were similar to those reported previously (Lagorce et al., 1998). This may be because of the different metabolism characteristics of clopidogrel in Han Chinese subjects.

Clopidogrel, an antiplatelet drug, has a favorable preventive effect against atherosclerosis-related diseases (Nyiro et al., 2012). In clinical practice, aspirin and clopidogrel represents the mainstay of antiplatelet treatment for preventing thromboembolic events in patients with ischemic cardiovascular diseases (Bauer et al., 2011). Clopidogrel is associated with high interpatient variability in efficacy and adverse reactions. A previous study indicated that $4-30 \%$ patients showed unsatisfactory antiplatelet effects after routine administration of clopidogrel, which resulted in a higher incidence of cardiovascular adverse events (i.e., recurrent myocardial infarction, thrombosis, and death). Recent studies showed that interpatient variability in efficacy and side effects were closely related to human gene polymorphisms (Terpening, 2010). Clopidogrel is a prodrug that requires activation by the cytochrome P450 enzymes (Johansson and Ingelman-Sundberg, 2011). CYP2C19 is an important isoenzyme required for the activation of clopidogrel. A previous study showed that individuals with a loss-of-function 
allele had a 1.5-2-fold greater risk of cardiovascular adverse events and deaths related to cardiovascular diseases than those without the allele (Damani and Topol, 2010). In addition, a previous study has shown the relationship between the presence of the loss-of-function allele (particularly CYP2C19*2) and the incidence of ischemic cardiovascular adverse events after administration of clopidogrel (Kreutz et al., 2010).

\section{REFERENCES}

Bauer T, Bouman HJ, van Werkum JW, Ford NF, et al. (2011). Impact of CYP2C19 variant genotypes on clinical efficacy of antiplatelet treatment with clopidogrel: systematic review and meta-analysis. BMJ 343: d4588.

Damani SB and Topol EJ (2010). The case for routine genotyping in dual-antiplatelet therapy. J. Am. Coll. Cardiol. 56: 109-111.

Johansson I and Ingelman-Sundberg M (2011). Genetic polymorphism and toxicology - with emphasis on cytochrome p450. Toxicol. Sci. 120: 1-13.

Junior EA, Duarte LF, Vanunci MLP, Oliveira DA, et al. (2010). Comparative biological availability of clopidogrel formulation in healthy volunteers after a single dose administration. J. Bioequiv Availab 2: 45-49.

Kreutz RP, Stanek EJ, Aubert R, Yao J, et al. (2010). Impact of proton pump inhibitors on the effectiveness of clopidogrel after coronary stent placement: the clopidogrel Medco outcomes study. Pharmacotherapy 30: 787-796.

Lagorce P, Perez Y, Ortiz J, Necciari J, et al. (1998). Assay method for the carboxylic acid metabolite of clopidogrel in human plasma by gas chromatography-mass spectrometry. J. Chromatogr. B Biomed. Sci. Appl. 720: 107-117.

Nyiro G, Inczedy-Farkas G, Remenyi V, Gal A, et al. (2012). The effect of the CYP 2C19*2 polymorphism on stroke care. Acta. Physiol. Hung. 99: 33-39.

Pereillo JM, Maftouh M, Andrieu A, Uzabiaga MF, et al. (2002). Structure and stereochemistry of the active metabolite of clopidogrel. Drug Metab. Dispos. 30: 1288-1295.

Savi P, Herbert JM, Pflieger AM, Dol F, et al. (1992). Importance of hepatic metabolism in the antiaggregating activity of the thienopyridine clopidogrel. Biochem. Pharmacol. 44: 527-532.

Stanisstreet M and Smith JL (1977). The effect of concanavalin-A on the reaggregation of cells dissociated from Xenopus laevis early embryos. Experientia 33: 1099-1100.

Terpening C (2010). Clopidogrel: a pharmacogenomic perspective on its use in coronary artery disease. Clin. Med. Insights Cardiol. 4: 117-128. 